\title{
AVALIAÇÃo DA ESTABILIDADE DO MARCADOR PLASMÁTICO DO ESTRESSE OXIDATIVO - MALONDIALDEÍDO
}

\author{
Denise Grotto, Juliana Valentini, Silvana Boeira, Clóvis Paniz, Lucas Santa Maria, Juliana Vicentini, Angela Moro, \\ Mariele Charão e Solange Cristina Garcia* \\ Departamento de Análises Clínicas e Toxicológicas, Universidade Federal de Santa Maria, CP 5061, 97110-970 Santa Maria - \\ RS, Brasil \\ Simone Gonçalves Cardoso \\ Departamento de Farmácia Industrial, Universidade Federal de Santa Maria, CP 5061, 97110-970 Santa Maria - RS, Brasil
}

Recebido em 15/1/07; aceito em 2/8/07; publicado na web em 19/12/07

\begin{abstract}
EVALUATION OF THE STABILITY OF THE OXIDATIVE STRESS PLASMATIC BIOMARKER - MALONDIALDEHYDE. Malondialdehyde (MDA) is one of the lipid peroxidation products widely used as indicator of cellular injury. However, the short-term and the long-term stability of this biomarker remain unclear. The objective of this work was to evaluate the stability of plasmatic MDA at $-20^{\circ} \mathrm{C}$, utilizing thiobarbituric acid (TBA) as derivative in spectrophotometric and chromatographic analysis. The results showed that MDA was stable for $24 \mathrm{~h}$ after blood collection, was not stable when stored after alkaline hydrolysis, remained stable for 30 days after TBA derivatization and was stable for 3 days when stored after n-butanol extraction, all at $-20^{\circ} \mathrm{C}$.
\end{abstract}

Keywords: oxidative stress; malondialdehyde; stability.

\section{INTRODUÇÃO}

As espécies reativas de oxigênio e de nitrogênio são produzidas de forma contínua pelas células como parte de seus processos metabólicos ${ }^{1}$. O desequilíbrio entre a formação e a remoção destas espécies, decorrente da diminuição dos antioxidantes endógenos ou ainda do aumento da geração de espécies oxidantes, gera um estado próoxidante. Este processo favorece a ocorrência de ataques dessas espécies reativas a componentes celulares, especialmente os lipídeos ${ }^{2}$. Assim, a peroxidação lipídica provoca dano tecidual, o qual está relacionado com a patogênese de várias doenças, entre elas aterosclerose ${ }^{3,4}$, diabetes ${ }^{4}$, doenças renais ${ }^{5}$ e Alzheimer $^{6}$.

Muitos biomarcadores vêm sendo utilizados para avaliar o estresse oxidativo, dentre eles malondialdeído $(\mathrm{MDA})^{7}$, dienos conjugados ${ }^{8}$, gases etano e pentano ${ }^{9}$, isoprostanos ${ }^{10}$, 4-hidroxi-nonenal (4-HNE) $)^{11}$, modificações de proteínas ${ }^{12}$ e modificações no DNA ${ }^{13}$. O MDA, porém, é um dos biomarcadores mais utilizados por ser um dos produtos secundários da peroxidação lipídica mais conhecidos ${ }^{11}$.

No entanto, os biomarcadores do estresse oxidativo são geralmente muito instáveis. Dessa forma, a análise confiável destes biomarcadores em amostras biológicas é muito importante, uma vez que resultados falsamente alterados podem ser gerados se as análises não forem realizadas sob condições analíticas adequadas. A influência do tempo de processamento das amostras, por exemplo, sobre a estabilidade do MDA não é muito conhecida, e deve ser bem estabelecida antes da sua aplicação na análise de amostras sanguíneas humanas, pois se objetivam resultados confiáveis e reprodutíveis na rotina laboratorial.

Além disso, o emprego de uma metodologia específica é de grande importância na análise laboratorial do MDA. O principal método utilizado é a reação do MDA com o ácido tiobarbitúrico (TBA) ${ }^{14}$, formando um complexo colorido que pode ser quantificado por espectrofotometria ou por cromatografia líquida de alta eficiência (CLAE), ambos com detecção visível. Uma vez que outros aldeídos podem reagir com o $\mathrm{TBA}^{15}$, a quantificação de MDA por

*e-mail: sgarpom@smail.ufsm.br espectrofotometria vem recebendo muitas críticas, e a cromatografia, por se tratar de uma técnica de separação, tem sido a técnica de escolha para a quantificação do MDA.

Neste trabalho, a estabilidade de MDA em plasma humano, em diferentes etapas de seu processamento, bem como a estabilidade do padrão aquoso de MDA foi verificada utilizando a técnica de CLAE com detecção visível, de acordo com o método de Grotto et $a l .{ }^{16}$. Além disso, a metodologia conhecida como TBARS ${ }^{17}$ (substâncias reativas ao ácido tiobarbitúrico) também foi utilizada em um estudo comparativo entre as metodologias, uma vez que o tratamento da amostra plasmática é diferente.

\section{PARTE EXPERIMENTAL}

\section{Reagentes}

Metanol e n-butanol grau HLPC foram comprados da Tedia Company (Fairfield USA). Ácido tiobarbitúrico, bis-malonildialdeído-dimetil-acetal e 2,6-di-tert-butyl-4-methylphenol (BHT) foram comprados de Sigma-Aldrich (St. Louis, MO, USA). Todos os outros reagentes usados eram de grau analítico. Soluções aquosas foram preparadas empregando água purificada através do sistema Milli-Q (Millipore, Bedford, MA, USA).

\section{Metodologia}

A quantificação do MDA em amostras de plasma foi realizada por meio de cromatografia líquida de alta eficiência (CLAE), com detecção visível (VIS), segundo a metodologia de Grotto et al. ${ }^{16}$. Além disso, a técnica de TBARS - VIS foi realizada, segundo a metodologia de Ohkawa et al. ${ }^{17}$ modificada, para o estudo comparativo dos níveis de MDA nas diferentes metodologias.

\section{Instrumentação e condições cromatográficas}

O equipamento utilizado foi o sistema cromatográfico Knauer®, 
modelo WellChrom, com bomba quaternária, reservatório de solventes, misturador, degaseificador para solventes, injetor manual com amostrador de volume fixo de $20 \mu \mathrm{L}$ e detector UV-VIS. O controle cromatográfico, a coleta e o processamento dos dados foram realizados usando o programa Eurochrom 2000 Software ${ }^{\circledR}$, edição básica 2.05 para Windows.

A separação cromatográfica foi feita utilizando coluna de fase reversa C18 Eurospher-100 150 x $4 \mathrm{~mm}$ com poro de $5 \mu \mathrm{m}$ de tamanho e pré-coluna Eurospher-100 5 x $4 \mathrm{~mm}$ com poro de $5 \mu \mathrm{m}$ de tamanho.

A fase móvel consistiu-se de uma mistura de $2,5 \mathrm{mM}$ de $\mathrm{KH}_{2} \mathrm{PO}_{4}$ - pH 7,0 e Metanol (50:50 v/v), eluição isocrática com fluxo de 0,6 $\mathrm{mL} / \mathrm{min}$. A absorbância do eluente foi monitorada a $532 \mathrm{~nm}$ e o tempo total de análise foi de $8 \mathrm{~min}$. A coluna foi aquecida a $40{ }^{\circ} \mathrm{C}$ pelo sistema termostatizado Chromacon ${ }^{\circledR}$.

Para a técnica de TBARS, o equipamento utilizado foi o espectrofotômetro Hitachi U-1800.

\section{Preparação das amostras}

\section{Soluções padrão}

Como padrão de MDA, utilizou-se o bis-malonildialdeídodimetil-acetal. Após sua diluição em água ultra pura, alíquotas de $200 \mu \mathrm{L}$ de solução de MDA na concentração de $3 \mathrm{mM}$ - solução estoque - foram armazenadas em tubos plásticos tipo "eppendorfs" a $-20{ }^{\circ} \mathrm{C}$. A estabilidade da solução estoque foi verificada durante 18 meses.

As soluções de trabalho foram preparadas diariamente, e obtidas por diluição da solução estoque $3 \mathrm{mM}$, com estabilidade verificada ao longo de um dia. Curvas analíticas de calibração, aquosas e com adição de padão na matriz biológica, foram preparadas nas concentrações de 0,$28 ; 0,56 ; 1,7 ; 3,4$ e $6,6 \mu \mathrm{M}$, de acordo com a metodologia validada por Grotto et al. ${ }^{16}$. A quantificação de MDA presente na própria amostra plasmática antes da adição do padrão foi considerada como nível basal.

\section{Coleta de sangue}

As amostras de sangue foram coletadas, após consentimento dos participantes, por venopunção, de 6 indivíduos sadios, com idade entre 20 e 25 anos, em tubos contendo EDTA como anticoagulante. As amostras foram mantidas em banho de gelo até o momento da centrifugação, a qual foi realizada em centrífuga refrigerada $\left(4{ }^{\circ} \mathrm{C}\right)$ a $1500 \mathrm{~g}$ por $10 \mathrm{~min}$, logo após a coleta.

\section{Procedimento para análise de MDA}

\section{Por cromatografia líquida (CLAE - VIS)}

Após centrifugação, o plasma foi separado. A um volume de 75 $\mu \mathrm{L}$ de plasma foram adicionados $25 \mu \mathrm{L}$ de água e $25 \mu \mathrm{L}$ de $\mathrm{NaOH}$ $3 \mathrm{~N}$, e incubados por $30 \mathrm{~min}$, em sistema de agitação horizontal com aquecimento a $60{ }^{\circ} \mathrm{C}$. Depois disto, $125 \mu \mathrm{L}$ de $\mathrm{H}_{3} \mathrm{PO}_{4} 6 \%$ e $125 \mu \mathrm{L}$ de TBA $0,8 \%$ foram adicionados às amostras e a mistura foi incubada por $45 \mathrm{~min}$ a $90{ }^{\circ} \mathrm{C}$. O próximo passo foi adicionar $50 \mu \mathrm{L}$ de dodecil sulfato de sódio (SDS) $10 \%$ às amostras. Em seguida, as amostras foram extraídas com $300 \mu \mathrm{L}$ de n-butanol após 1 min de vórtex, e centrifugadas a $3000 \mathrm{~g}$ por $10 \mathrm{~min}$. Alíquotas de $20 \mu \mathrm{L}$ da fase orgânica foram injetadas no cromatógrafo.

\section{Por espectrofotometria - TBARS}

A um volume de $200 \mu \mathrm{L}$ de plasma foram adicionados $1100 \mu \mathrm{L}$ de $\mathrm{H}_{3} \mathrm{PO}_{4} 1,4 \%, 20 \mu \mathrm{L}$ de BHT (diluído em etanol) e $500 \mu \mathrm{L}$ de TBA $0,6 \%$. As amostras foram incubadas por $45 \mathrm{~min}$ a $90{ }^{\circ} \mathrm{C}$. Posteriormente, $200 \mu \mathrm{L}$ de SDS $8,7 \%$ foram adicionados às amostras, as quais foram homogeneizadas, centrifugadas a $3000 \mathrm{~g}$ por 10 min e lidas em espectrofotômetro em comprimento de onda de 532 nm.

\section{Protocolo elaborado para o estudo da estabilidade da amostra biológica}

O estudo da estabilidade do MDA nas amostras de plasma, por CLAE - VIS, seguiu-se da seguinte forma: imediatamente após a coleta do sangue, uma amostra de plasma foi processada em duplicata e quantificada (dia zero); alíquotas de plasma foram separadas em ependorffs, armazenadas a $4{ }^{\circ} \mathrm{C}$, e analisadas $24 \mathrm{~h}$ após a coleta do sangue; alíquotas de plasma foram separadas em eppendorfs, armazenadas a $-20^{\circ} \mathrm{C}$ e analisadas nos dias $1,2,3$ e 4 após a coleta do sangue, com e sem a adição do antioxidante BHT; alíquotas de plasma sofreram hidrólise alcalina e foram imediatamente armazenadas a $-20^{\circ} \mathrm{C}$. Após os dias 1 e 2 , o protocolo foi seguido até as análises cromatográficas; alíquotas de plasma, depois da hidrólise alcalina, foram derivatizadas com TBA e estocadas a $-20^{\circ} \mathrm{C}$. Após os dias 5, 10, 20, 30 e 35, o protocolo foi seguido até as análises cromatográficas; finalmente, a reação foi desenvolvida por completo e as amostras extraídas com n-butanol foram armazenadas a $-20^{\circ} \mathrm{C}$. A estabilidade foi avaliada nos dias $1,2,3 \mathrm{e}$ 4.

Além disso, para a comparação da estabilidade das amostras plasmáticas pelo método de TBARS, que não utiliza a hidrólise alcalina, alíquotas de plasma foram analisadas no momento da coleta, enquanto outras foram armazenadas a $-20{ }^{\circ} \mathrm{C}$ e avaliadas $24 \mathrm{e}$ $48 \mathrm{~h}$ após o armazenamento. Também se investigou a estabilidade após a derivatização das amostras plasmáticas com TBA nos tempos zero, $1,5,10$ e 20 dias após armazenamento a $-20{ }^{\circ} \mathrm{C}$.

\section{Análise estatística}

Os resultados foram expressos como média \pm desvio padrão. A análise estatística foi realizada através do programa Statistica, aplicando-se o teste $\mathrm{t}$ de Student, $\mathrm{p}<0,05$ foi considerado significativo. Além disso, amostras com variação máxima de $15 \%$ foram consideradas estáveis, de acordo com a ANVISA ${ }^{18}$.

\section{RESULTADOS}

As curvas analíticas apresentaram, segundo a equação do gráfico, $r^{2}=0,9984$ e $r^{2}=0,9995$ para as curvas com adição de padrão em plasma e para a curva aquosa, respectivamente.

Com o presente estudo, pôde-se observar que a solução estoque de MDA $3 \mathrm{mM}$ mostrou-se estável a $-20{ }^{\circ} \mathrm{C}$ ao longo dos 18 meses em que foi analisada (Figura 1). Já com relação à estabilidade das soluções de trabalho de MDA, estas não se mostraram estáveis. Este fato foi verificado pelas curvas de calibração. Após $6 \mathrm{~h}$ da diluição da solução estoque de MDA, o ponto mais concentrado da curva, por exemplo, de $6,6 \mu \mathrm{M}$ decaía para $4,4 \mu \mathrm{M}$, com cerca de $30 \%$ de perda. Por isso, seu uso foi verossímil somente até $6 \mathrm{~h}$ após a diluição, quando estocadas a $-20{ }^{\circ} \mathrm{C}$. Quando eram armazenadas em banho de gelo, as soluções de trabalho de MDA mantinham sua estabilidade por no máximo $2 \mathrm{~h}$, sendo que a concentração de 6,6 $\mu \mathrm{M}$ decaía para 4,0 $\mu \mathrm{M}$.

No estudo da estabilidade do MDA com e sem a adição do antioxidante BHT, quantificado por CLAE-VIS, os resultados obtidos estão apresentados na Figura 2. Verificou-se que as amostras de plasma armazenadas a $-20{ }^{\circ} \mathrm{C}$ com BHT eram estáveis por 3 dias (variação $<15 \%$ ), resultado contrário às amostras armazenadas sem BHT, que se mostraram estáveis por somente $24 \mathrm{~h}$ (variação de $18 \%$ na área do pico no segundo dia). Porém, devido a estudos 


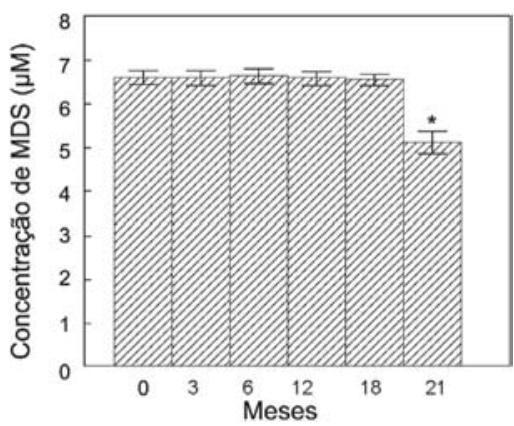

Figura 1. Análise da estabilidade da solução aquosa estoque de MDA, mostrada em meses, armazenadas a $-20^{\circ} \mathrm{C}$, ao longo do processo de quantificação do MDA, por CLAE - VIS. A concentração de MDA representa o ponto mais concentrado da curva analítica $(6,6 \mu M) *$

contestando o uso do BHT como antioxidante, testes foram realizados com amostras de plasma com BHT, sem BHT e com etanol, principal diluente do BHT. Os resultados obtidos foram de 2,55 \pm $0,18 \mu \mathrm{M}$ de MDA para as amostras com BHT, 4,60 $\pm 0,20 \mu \mathrm{M}$ de MDA para as amostras sem BHT e 2,58 $\pm 0,16 \mu \mathrm{M}$ de MDA para as amostras com etanol. Não se observou diferença significativa entra as amostras de plasma armazenadas com BHT e com etanol $(\mathrm{p}=0,1)$. Porém, verificou-se uma diminuição significativa dos níveis de MDA plasmático utilizando BHT comparado aos níveis sem BHT $(p=0,001)$.
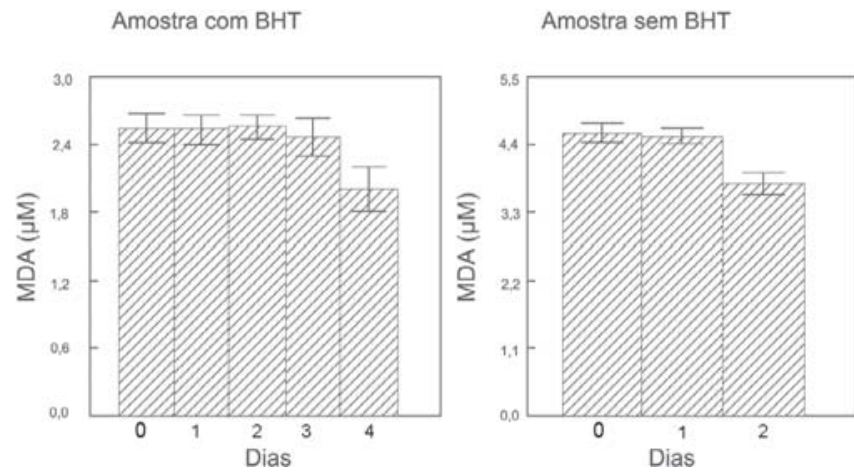

Figura 2. Análise da estabilidade dos níveis de MDA em amostras plasmáticas, com e sem o antioxidante BHT, mostrada em dias, realizado por CLAE - VIS e armazenados $a-20^{\circ} \mathrm{C}$. Uma diferença significativa entre os níveis de MDA das amostras com e sem BHT foi encontrada $(p=0,001)$

Quanto à estabilidade da amostra plasmática armazenada, a concentração inicial encontrada imediatamente após a coleta foi de 4,60 $\pm 0,15 \mu \mathrm{M}$, enquanto que após $24 \mathrm{~h}$, armazenada a $4{ }^{\circ} \mathrm{C}$, este nível passou para $8,74 \pm 0,5 \mu \mathrm{M}$, demonstrando uma variação de $90 \%$.

Os resultados do estudo da estabilidade do MDA nas outras etapas de processamento da amostra plasmática são mostrados na Tabela 1. Quando as amostras foram estocadas após o processo de hidrólise alcalina, observou-se, logo nas primeiras 24 h após o armazenamento, uma perda de cerca de 50\% nos níveis de MDA. Quando as amostras foram derivatizadas com TBA e armazenadas a $-20{ }^{\circ} \mathrm{C}$ se mostraram estáveis ao longo de 30 dias, com degradação de cerca de $20 \%$ do MDA após este período. Após a extração das amostras com n-butanol e armazenamento a $-20{ }^{\circ} \mathrm{C}$, estas permaneceram estáveis por 3 dias. Além disso, observou-se que entre o período de extração da amostra com n-butanol e sua injeção no cromatógrafo, estas deveriam permanecer em banho de gelo, pois à temperatura ambiente as amostras se degradavam.
Tabela 1. Análise da estabilidade dos níveis de MDA em amostras plasmáticas, armazenadas a $-20{ }^{\circ} \mathrm{C}$, ao longo do processo de quantificação do MDA, por CLAE - VIS

\begin{tabular}{lc}
\hline Amostra após hidrólise & Concentração de MDA $(\mu \mathrm{M})$ \\
\hline Dia zero & $4,60 \pm 0,15$ \\
Dia 1 & $2,38 \pm 0,20$ \\
Após derivatização com TBA & $4,60 \pm 0,15$ \\
Dia zero & $4,63 \pm 0,12$ \\
Dia 5 & $4,61 \pm 0,18$ \\
Dia 10 & $4,56 \pm 0,12$ \\
Dia 20 & $4,66 \pm 0,13$ \\
Dia 30 & $3,75 \pm 0,15$ \\
Dia 35 & \\
Após extração com n-butanol & $4,60 \pm 0,15$ \\
Dia zero & $4,60 \pm 0,18$ \\
Dia 1 & $4,56 \pm 0,12$ \\
Dia 2 & $4,53 \pm 0,18$ \\
Dia3 & $3,80 \pm 0,13$ \\
Dia 4 & \\
\hline
\end{tabular}

Na Tabela 2, são apresentados os resultados comparativos entre as duas metodologias, pois além de utilizar técnicas de quantificação diferentes, CLAE-VIS e TBARS, uma realiza hidrólise e a outra não. Observa-se uma diferença significativa entre os níveis de MDA quantificados por CLAE quando comparada à espectrofotometria $(\mathrm{p}<0,001)$. Ambas as metodologias demonstraram que as amostras de plasma permaneceram estáveis por $24 \mathrm{~h}$ a $-20^{\circ} \mathrm{C}$. Na etapa de derivatização com TBA, porém, as amostras que tiveram o MDA quantificado por CLAE - VIS mostraram-se estáveis por 30 dias, enquanto que o MDA quantificado por TBARS foi estável por 20 dias.

Tabela 2. Comparação da estabilidade dos níveis de MDA em amostras de plasma armazenadas a $-20{ }^{\circ} \mathrm{C}$ logo após coleta e após derivatização com TBA, realizadas pelas técnicas de CLAE - VIS e TBARS. Uma diferença significativa entre os níveis de MDA quantificados por CLAE e por espectrofotometria foi encontrada $(\mathrm{p}<0,001)$

\begin{tabular}{lccc}
\hline Tratamento & Tempo (dias) & CLAE - VIS & TBARS \\
\hline Armazenamento & 0 & $4,6 \pm 0,15$ & $6,90 \pm 0,31$ \\
logo após coleta & 1 & $4,55 \pm 0,11$ & $7,10 \pm 0,26$ \\
& 2 & $3,91 \pm 0,18$ & $7,50 \pm 0,25$ \\
Armazenamento & 0 & $4,6 \pm 0,15$ & $6,80 \pm 0,71$ \\
após derivatização & 1 & $4,61 \pm 0,14$ & $6,90 \pm 0,58$ \\
com TBA & 5 & $4,61 \pm 0,12$ & $7,20 \pm 0,44$ \\
& 10 & $4,64 \pm 0,18$ & $7,55 \pm 0,85$ \\
& 20 & $4,70 \pm 0,20$ & $7,50 \pm 0,60$ \\
& 30 & $4,62 \pm 0,13$ & $8,10 \pm 0,83$ \\
& 35 & $3,75 \pm 0,15$ & \\
\hline
\end{tabular}

\section{DISCUSSÃO}

Uma vez que a realização de um estudo de estabilidade é de grande relevância para a rotina clínica laboratorial, com este trabalho pôde-se verificar, em diferentes etapas do processamento das amostras de plasma, o armazenamento e a estabilidade do biomarcador malondialdeído.

Observando-se os resultados, é possível perceber a baixa estabilidade das soluções aquosas de trabalho de MDA. Estáveis por no máximo $2 \mathrm{~h}$ em banho de gelo, estas soluções devem ser manipuladas logo após sua diluição, evitando assim a perda de MDA, principalmente 
quando curvas de calibração são feitas. Por outro lado, a solução estoque de MDA mostrou ter um bom tempo de estabilidade a $-20{ }^{\circ} \mathrm{C}$, podendo ser utilizada por até 18 meses, sem qualquer perda.

$\mathrm{O}$ uso do BHT como um antioxidante poderia prevenir a peroxidação lipídica durante os procedimentos ${ }^{7}$. Porém, o BHT é dissolvido em etanol ou metanol, solventes que podem ser utilizados como desproteinizantes. Pilz e colaboradores ${ }^{19}$ verificaram que a solução etanólica por si só reduzia os valores de MDA por provocar uma desproteinização antes que ocorresse a hidrólise das proteínas, levando a uma perda de MDA por co-precipitação, pois se sabe que uma parte do MDA se encontra ligado às proteínas plasmáticas. No presente estudo, testes com e sem o BHT e somente com o etanol foram realizados. Os níveis plasmáticos de MDA encontrados tanto para as amostras com BHT quanto para as amostras somente com etanol não foram significativamente diferentes $(\mathrm{p}=0,1)$. Além disso, os resultados se apresentaram significativamente diminuídos quando comparados aos níveis de MDA sem BHT $(p=0,001)$. Assim, a adição de BHT nas amostras de plasma pode contribuir para obtenção de resultados falsamente diminuídos. O volume de amostra utilizado por Grotto et al. ${ }^{16}$ é de apenas $75 \mu \mathrm{L}$ de plasma e uma adição de $25 \mu \mathrm{L}$ de BHT etanólico pode desproteinizar a amostra antes que todo o MDA seja hidrolisado, alterando de maneira ilusória os valores reais deste biomarcador. Por outro lado, analisando-se somente a questão estabilidade da amostra plasmática, observou-se que utilizando BHT a quantificação pode ser realizada até 3 dias, sem perda considerável $(<15 \%)$, quando a amostra é armazenada a $-20{ }^{\circ} \mathrm{C}$. No entanto, neste trabalho não se adotou o uso de BHT por entender que este possa contribuir na obtenção de resultados falsamente diminuídos e, talvez, a solução seja procurar um antioxidante, evitando a perda por coprecitação do MDA.

Quando armazenadas a $4{ }^{\circ} \mathrm{C}$, as amostras plasmáticas não se mostraram estáveis. Isto ocorreu, provavelmente, pela peroxidação dos ácidos graxos polinsaturados in vitro, elevando os níveis de MDA erroneamente, demonstrando que as amostras, nesta condição de armazenamento, não mantêm sua estabilidade.

Com relação à estabilidade das amostras de plasma, quantificadas por CLAE-VIS, os resultados encontrados servem como referência, uma vez que são poucos os trabalhos abordando este importante parâmetro ${ }^{20}$. As amostras de plasma armazenadas a $-20{ }^{\circ} \mathrm{C}$ logo após a coleta mostraram-se estáveis somente nas primeiras $24 \mathrm{~h}$. Com isso, é importante lembrar os cuidados que se deve ter em estudos epidemiológicos para que não haja uma falsa alteração dos níveis de MDA, causada pela demora no processamento da amostra. Wu e colaboradore ${ }^{20}$ observaram que o MDA não se mostrou estável após armazenamento em gelo em 24 e $36 \mathrm{~h}$. Além disso, muitos trabalhos citam o armazenamento da amostra de plasma a $-20{ }^{\circ} \mathrm{C}$ logo após a coleta, contudo, o tempo de armazenagem não é constatado ${ }^{21-23}$. Wong e colaboradores ${ }^{24}$ observaram que as amostras biológicas não devem ser armazenadas por mais de $24 \mathrm{~h}$ a $4{ }^{\circ} \mathrm{C}$.

As amostras de plasma armazenadas após a etapa de hidrólise alcalina não se mostraram estáveis, uma vez que há perda do MDA e seus valores chegam a diminuir $50 \%$ já no primeiro dia após o armazenamento.

Por outro lado, as amostras de plasma armazenadas após a derivatização com o TBA podem ser quantificadas por até 30 dias após o armazenamento, quando quantificadas pelo método de Grotto et al. ${ }^{16}$ e até 20 dias quando quantificadas pelo método de Ohkawa et al. ${ }^{17}$. Este fato é de grande relevância em estudos que abrangem um grande número de indivíduos, visto que as amostras devem ser processadas no momento da coleta, tendo-se, porém, um período de tempo relativamente longo para se realizar as quantificações cromatográficas ou espectrofotométricas com a segurança de que os valores encontrados representarão os valores reais.

No estudo da estabilidade comparativo entre as duas metodologias, houve um aumento significativo nos níveis de MDA quantificado por espectrofotometria comparado aos níveis de MDA quantificado por CLAE-VIS. Esta diferença comprova que a metodologia de TBARS não é específica, e que outras substâncias ${ }^{15}$ reagem com o TBA.

Além disso, os valores de MDA quantificados pela técnica de TBARS apresentaram aumento de seus níveis após 20 dias. Este fato pode ser explicado, provavelmente, pela presença de grande quantidade de interferentes presentes na amostra quantificada por TBARS, uma vez que a metodologia de Ohkawa et al. ${ }^{17}$ modificada não emprega hidrólise alcalina, o que poderia influenciar na instabilidade obtida, bem como no aumento da presença de interferentes.

\section{CONCLUSÃO}

Sabendo-se da relação da peroxidação lipídica com diferentes patologias, a quantificação laboratorial confiável do biomarcador malondialdeído como um indicador do estresse oxidativo é de grande importância. Além disso, tendo em vista o pequeno número de trabalhos que relacionam o armazenamento e a estabilidade deste biomarcador, uma padronização da estabilidade do MDA em amostras de plasma em diferentes etapas de sua reação com TBA foi obtida com o presente estudo.

Neste trabalho, estabeleceu-se que as amostras de plasma, armazenadas a $4{ }^{\circ} \mathrm{C}$ não são estáveis e que a $-20{ }^{\circ} \mathrm{C}$ podem ser mantidas por até $24 \mathrm{~h}$ sem BHT. Uma vez realizada a derivatização, as amostras mostraram possibilidade de quantificação sem perdas consideráveis por até 20 dias utilizando-se a espectrofotometria e de até 30 dias utilizando-se a cromatografia. Assim, os resultados do método utilizando CLAE-VIS com hidrólise alcalina foram estáveis por maior tempo.

O método utilizando espectrofotometria apresentou resultados mais elevados comparados aos obtidos por CLAE-VIS, provavelmente devido à inespecificidade do primeiro, pois se sabe que o TBA reage com outros aldeídos além do MDA.

Diante dos resultados mostrados neste trabalho, é importante salientar a necessidade de se adotar condições analíticas idênticas quando trabalhos técnico-científicos são realizados, priorizando assim o tempo e acondicionamento das amostras biológicas, garantindo resultados confiáveis, sem prejuízo ao diagnóstico clínico, independente da técnica ou metodologia utilizada.

\section{AGRADECIMENTOS}

Ao CNPq e à FAPERGS pelos auxílios financeiros; à Knauer ${ }^{\circledR}$ pelo suporte do aparelho de cromatografia líquida de alta eficiência. Aos órgãos de fomento CAPEs, CNPq e FAPERGS pelas bolsas de mestrado e iniciação científica concedidas, e pela bolsa de pesquisadora do CNPq à Dra. S. C. Garcia.

\section{REFERÊNCIAS}

1. Urso, M. L.; Clarkson, P. M.; Toxicology 2003, 189, 41.

2. Gillham, B.; Papachristodoulou, D. K.; Thomas, J. H.; Wills': Biochemical basis of medicine, $3^{\text {rd }}$ ed., Butterworth- Heinemann: Oxford, 1997.

3. Halliwell, B.; Br. Med. J.. 1993, 307, 885.

4. Kesavulu, M. M.; Rao, B. K.; Giri, R.; Vijaya, J.; Subramanyam, G.; Apparao, C.; Diabetes Research and Clinical Practice 2001, 53, 33.

5. Scott, B.; Deman, A.; Peeters, P.; van den Branden, C.; Stolear, J. C.; van Camp, G.; Verbrrlen, D.; Nephrol. Dial. Transplant. 2003, 18, 737.

6. Joosten, E.; Clin. Chem. Lab. Med. 2001, 39, 717.

7. Esterbauer, H.; Cheeseman, K. H.; Meth. Enzymol. 1990, 186, 407.

8. Dormandy, T. L.; Wickens, D. G.; Chem. Phys. Lipids 1987, 45, 356. 
9. Frank, H.; Hintze, T.; Bimboes, D.; Remmer, H.; Toxicol. Appl. Pharmacol. 1980, 56, 337.

10. Morrow, J. D.; Frei, B.; Longmire, A. W.; Gaziano, J. M.; Lynch, S. M.; Shyr, Y.; Strauss, W. E.; Oates, J. A.; Roberts, L. J.; N. Engl. J. Med. 1995, 332, 1198.

11. Esterbauer, H.; Schaur, R. J.; Zollner, H.; Free Radical Biol. Med. 1991 11,81 .

12. Levine, R. L.; Williams, J. A.; Stadtman, E. R.; Shacter, E.; Meth. Enzymol. 1994, 233, 346

13. Marnett, L. J.; Mutat. Res. 1999, 424, 83

14. Yagi, K.; Nishigaki, I.; Ohama, H.; Vitamins 1968, 37, 105.

15. Sinnhuber, R. O.; Yu, T. C.; J. Jpn. Oil Chemist's Soc. 1977, 26, 259.

16. Grotto, D.; Santa Maria, L. D.; Boeira, S.; Valentini, J.; Charão, M. F.; Moro, A. M.; Nascimento, P. C.; Pomblum, V. J.; Garcia, S. C.; J. Pharm. Biomed. Anal. 2007, 43, 619.

17. Ohkawa, H.; Ohishi, N.; Anal. Biochem. 1979, 95, 351.
18. ANVISA - Agência Nacional de Vigilância Sanitária, Brasil; Resolução $n^{\circ} 899$, de 29 de maio de 2003. Determina a publicação do guia para validação de métodos analíticos e bioanalíticos. Diário Oficial da República Federativa do Brasil, disponível em www.anvisa.gov.br/legis/resol/ 2003.htm

19. Pilz, J.; Meineke, I.; Gleiter, H. C.; J. Chromatogr., B: Anal. Technol. Biomed. Life Sci. 2000, 742, 315.

20. Wu, T.; Rifai, N.; Roberts II, L. J.; Willett, W. C.; Rimm E. B.; Cancer Epidemiol. Biomarkers Prev. 2004, 13, 1399.

21. Lykkesfeldt, J.; Clin. Chem. 2001, 47, 1725.

22. Sim, A. S.; Salonikas, C.; Naidoo, D.; Wilcken, D. E.; J. Chromatogr., B: Anal. Technol. Biomed. Life Sci. 2003, 785, 337.

23. Hong, Y.; Yeh, S.; Chang, C.; Hu, M.; Clin. Biochem. 2000, 33, 619.

24. Wong, S. H.; Knight, J. A.; Hopfer, S. M.; Zaharia, O.; Leach, C. N.; Sunderman, F. W.; Clin. Chem. 1987, 33, 214 